\title{
How to Identify White Coat Hypertension at The First Diagnosis: Establishment of A Scoring Model for The Differential Diagnosis of White Coat Hypertension and Sustained Hypertension
}

\section{Peng Cai}

Army Medical University https://orcid.org/0000-0001-8329-2528

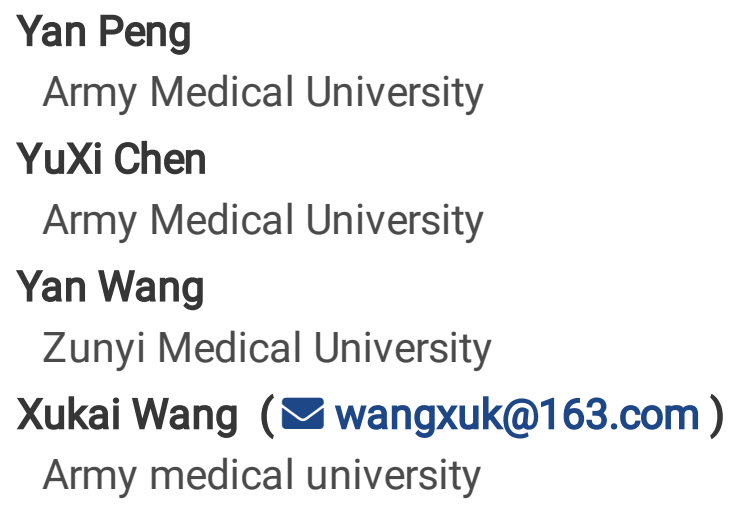

\section{Research}

Keywords: White coat hypertension, sustained hypertension, scoring model, nomogram

Posted Date: August 13th, 2020

DOI: https://doi.org/10.21203/rs.3.rs-49923/v1

License: (c) (1) This work is licensed under a Creative Commons Attribution 4.0 International License. Read Full License 


\section{Abstract}

Background: To establish a scoring model for the differential diagnosis of white coat hypertension (WCH) and sustained hypertension (SHT).

Methods: 553 adults with elevated office blood pressure, normal renal function and no antihypertensive drugs were included in this study. 17 parameters, such as gender and age, were obtained by questionnaire investigation and biochemical index detection. WCH and SHT were distinguished by 24-hour ambulatory blood pressure monitoring. Participants were randomly divided into a training set (445 cases) and a verification set (108 cases). In the training set, the above parameters were screened by LASSO regression and univariate logistic regression analysis, then, the scoring model was constructed through multivariate logistic regression analysis. ROC curve and calibration curve were used to discuss the discrimination and calibration of this scoring model respectively

Results: 6 parameters were finally selected, namely isolated systolic hypertension, systolic blood pressure, diastolic blood pressure, triglyceride, serum creatinine, and cardiovascular and cerebrovascular diseases. Multivariate logistic regression was used to establish the scoring model. The $\mathrm{R}^{2}$ and $A U C$ of the scoring model in the training set were 0.163 and 0.705 , respectively. In the verification set, the $R^{2}$ of the scoring model was 0.206 , and AUC was 0.718 . The calibration test results showed that the scoring model had good stability in both training set and verification set $(M S E=0.001, M A E=0.014 ; M S E=0.001$, $M A E=0.025$, respectively).

Conclusion: A stable scoring model for distinguishing WCH can be established, which can assist clinical medical workers to identify $\mathrm{WCH}$ at the first diagnosis.

\section{Background}

In the clinical practice, the differential diagnosis of white coat hypertension (WCH) and sustained hypertension (SHT) is of great significance. Both WCH and SHT are highly prevalent diseases, but the target organ damage effects of WCH and SHT are far from each other [1,2]. SHT is a hypertension subtype with definite damage to various target organs [3]. However, whether WCH is only a benign clinical phenomenon or a criminal clinical disease is still controversial [4]. Especially since the European society of hypertension practice guidelines (2014 edition) revised the diagnostic criteria for $\mathrm{WCH}$ and excluded isolated nocturnal hypertension with definite target organ injury effect from $\mathrm{WCH}$, the definite cardiovascular and cerebrovascular injury effect of WCH has not been found in many clinical studies [5, 6]. For example, a large-scale clinical study including 115,708 samples in 2017 did not find cardiovascular injury of $\mathrm{WCH}$ with normal ambulatory blood pressure in each time period [7]. Both WCH and SHT showed elevated office blood pressure, but the effects of cardiovascular and cerebrovascular injury were significantly different [8]. Reasonable discrimination between WCH and SHT in the clinical practice is helpful for medical personnel to understand the blood pressure status of patients more comprehensively and provide more reasonable treatment schemes. 
Although the clinical identification of WCH and SHT is so important, the initial identification of WCH and SHT has always been a difficult problem in the clinical practice [9]. At present, the identified methods include home blood pressure measurement and ambulatory blood pressure monitoring [10, 11]. At present, home self-test blood pressure is not popular, the method of measuring home blood pressure for the general population is not standardized, and home self-test blood pressure cannot reflect the night blood pressure level, and its differential effect between WCH and SHT is lower than ambulatory blood pressure monitoring [12]. However, the clinical application of 24-hour ambulatory blood pressure monitoring also requires newly diagnosed patients to spend more time and cost, especially it takes more than 24 hours to identify WCH and SHT when returning to the clinic, which is unacceptable to some patients. If medical personnel can predict the $\mathrm{WCH}$ risk of patients with increased office blood pressure during the initial diagnosis, ambulatory blood pressure monitoring can be selectively carried out, and clinical treatment can be carried out more reasonably.

Some medical workers empirically distinguish patients with elevated office blood pressure in clinical practice to judge whether WCH exists. Of course, this is based on the fact that clinicians have a certain understanding of the clinical characteristics of $\mathrm{WCH}$ and SHT. Previous literature reports suggest that WCH is often high in women, and clinically characterized by isolated systolic hypertension (ISH), but the incidence rate of $\mathrm{WCH}$ is low among smokers, while SHT shows more obvious blood lipid and renal function abnormalities, and is more prone to cardiovascular and cerebrovascular diseases [13-15]. Some medical personnel make an empirical evaluation of WCH and SHT based on known clinical characteristics, but there is a lack of a scientific quantitative scoring method. The gradual application of the scoring model for differential diagnosis in recent years has provided it with the possibility. The scoring model belongs to the risk prediction models. Its outstanding advantage is that it can combine several differential variables through the regression equation to improve the identification ability, and it can vividly measure the disease risk by drawing nomogram $[16,17]$. The purpose of this study is to construct a scoring model for differential diagnosis of WCH and SHT through the least absolute shrinkage and selection operator (LASSO) regression, univariate and multivariate logistic regression analysis and other statistical methods, and hope to apply it to clinical practice.

\section{Methods}

\section{Study participants}

Between April 2018 and June 2020, 826 adults with elevated office blood pressure were recruited as participants in Daping Hospital. Participants were further screened through the following two exclusion criteria: (1) antihypertensive drug users; (2) renal insufficiency ( $\mathrm{Scr} \geq 133 \mathrm{~mol} / \mathrm{L}$ ) [18]. The study was reviewed and approved by the Ethics Committee of Daping Hospital of the Army Medical University and registered in the Chinese Clinical Trial Registry with the registration number ChiCTR1800015507. All participants gave informed consent to the study.

\section{General clinical Information}


The age, sex, smoking history, drinking history, diabetes history, and cardiovascular and cerebrovascular disease history of the participants were collected through questionnaires. The height and weight of the selected participants were measured, and the body mass index (BMI) was calculated. The calculation formula was $(\mathrm{BMI})=$ weight $(\mathrm{kg}) \div$ height $^{2}(\mathrm{~m})[19]$

\section{Blood pressure measurement}

After the participants took a seat rest in the clinic for 20 minutes, the blood pressure of the right brachial artery was measured 3 times by professional medical personnel with mercury sphygmomanometer. The average value of blood pressure measurement was taken as office blood pressure measurement result [20].

24-hour ambulatory blood pressure monitoring was carried out by ambulatory ECG blood pressure recorder CB-2301-A (Wuxi, China), with 6: 00-22: 00 as daytime blood pressure and 22: 00-6: 00 as nighttime blood pressure. Daytime blood pressure measurement is conducted every 30 minutes, the number of effective sphygmomanometers should be above $80 \%$, and nighttime blood pressure requires effective blood pressure every hour.

WCH and SHT were distinguished according to office blood pressure and 24-hour ambulatory blood pressure. European Hypertension Practice Guideline Standard (2014 Edition) was adopted, and the blood pressure rise boundary point was set at office blood pressure $\geq 140 / 90 \mathrm{mmHg}$. The average ambulatory blood pressure was $\geq 135 / 85 \mathrm{mmHg}$ during the daytime, $\geq 120 / 70 \mathrm{mmHg}$ during the nighttime, and $\geq$ $130 / 80 \mathrm{mmHg}$ during the whole day $[21,22]$. SHT was defined as elevated office blood pressure and ambulatory blood pressure. While, WCH only showed elevated office blood pressure [23].

\section{Biochemical detection}

The total cholesterol (TC), triglyceride (TG), high-density lipoprotein cholesterol (HDL-C), low-density lipoprotein cholesterol (LDL-C), fasting blood glucose (Glu), serum creatinine (Scr) and uric acid (UA) were measured by BECKMAN AU5800 biochemical analyzer. Diabetes mellitus (DM) is defined as the previous definite diagnosis of diabetes mellitus, or the fasting blood glucose detected this time is $\geq 7.0 \mathrm{mmol} / \mathrm{L}$ [24].

\section{Division of training set and verification set}

Using RStudio software (Version 1.3. 959), the participants were divided into the training set and the verification set, and the ratio of training set to verification set was 4:1. The statistical differences of age, sex, WCH prevalence rate, and other research variables between the training set and the verification set were compared. The differences of research variables between WCH patients and SHT patients in training set and verification set were also analyzed respectively. The counting data is expressed as the number of cases (\%), and the Pearson chi-square test is used for statistical analysis. The measurement data are 
expressed as median (IQR, interquartile range), and the statistical analysis method adopts Kruskal-Wallis rank-sum test $[25,26]$. The above statistical analysis was performed by SPSS (Version 22.0) software.

\section{Model construction}

LASSO regression and univariate logistic regression were used to select the variables of the model. The study variables involved in the screening process included sex, age, body mass index (BMI), smoking history, drinking history, isolated systolic hypertension (ISH), systolic blood pressure (SBP), diastolic blood pressure (DBP), heart rate (HR), diabetes mellitus (DM), total cholesterol (TC), triglyceride (TG), high-density lipoprotein cholesterol (HDL-C), low-density lipoprotein cholesterol (LDL-C), creatinine (Scr), uric acid (UA) and cardiovascular and cerebrovascular diseases (CCVD). Firstly, the least absolute shrinkage and selection operator (LASSO) regression model was constructed in the training set. LASSO regression algorithm, which is suitable for the regression of high-dimensional data and interactive data analysis, was conducted by 10 -fold cross-validation with penalty parameter tuning based on minimum criteria and 1 standard error of the minimum criteria (the 1-SE criteria) in the training set. Since increasing the number of independent variables of the model cannot significantly improve the performance of the model after the value reaches a certain value, the 1-SE criteria can give the model with excellent performance but the least number of independent variables, so we generally adopt this criteria [27]. We further carried out univariate logistic regression analysis on the research variables corresponding to the 1SE criteria, and included the research variables with statistical differences as the final model construction variables. Multivariate logistic regression analysis was used to construct the scoring model of $\mathrm{WCH}$, and the corresponding nomogram was drawn [28]. The R language packets used mainly include "readxl", "glmnet", "informationValue", "rms", "pROC" and so on.

\section{Discrimination test}

Firstly, the receiver operating curve (ROC) was used in the training set to test the discrimination degree of the scoring model in the training set, and the coefficient of determination $\left(R^{2}\right)$, the area under the ROC curve (AUC) and its $95 \%$ confidence interval $(95 \% \mathrm{Cl})$ are obtained. Furthermore, after applying the scoring model to the verification set, the ROC curve was used to evaluate the discrimination degree of the verification set model, and whether the discrimination degree was similar to the training set model was observed [29].

\section{Calibration degree test}

Firstly, the Bootstrap method was used to randomly select samples 1000 times in the training set to verify the calibration degree of the model. The corresponding Calibration curves are made, and the mean square error (MSE) and mean absolute error (MAE) values are calculated to evaluate the model. The lower the MAE and MSE, the better the stability of the model [30]. Furthermore, the calibration degree of the model in the verification set is also checked by the same method.

\section{Expand application}


European Hypertension Practice Guidelines (2018 Edition) recommend ambulatory blood pressure monitoring for patients with grade 1 hypertension (SBP of $140-159 \mathrm{mmHg}$ and/or DBP of 90-99 mm Hg) or hypertensive patients without target organ damage to screen WCH [31]. Then this study took patients with grade 1 hypertension and hypertensive patients without CCVD as the subjects for ambulatory blood pressure examination according to the guidelines, and the corresponding method was called the hypertension guideline method (Method 1). Based on our scoring model, we use participants with WCH risk higher than 0.2 as screening subjects for ambulatory blood pressure monitoring, and the corresponding method is considered as the scoring model method (Method 2). We further compared the difference between the sample participation rate and WCH missed diagnosis rate between the two methods. The formula for calculating the participation rate and missed diagnosis rate is as follows.

\section{Results}

A total of 826 adults with elevated office blood pressure volunteered to participate in this study. 241 participants who took antihypertensive drugs and 32 participants who had renal insufficiency ( $\mathrm{Scr} \geq 133$ $\mathrm{mol} / \mathrm{L}$ ) were excluded. Finally, 553 adults were included in the study, 304 males and 249 females, with an average age of 63.6 years.

\section{Training Set and verification Set}

Participants are randomly divided into the training sets and verification sets. There were 445 participants in the training set and 108 participants in the verification set. The prevalence rate of $\mathrm{WCH}$ in the training set was $35.1 \%$, and that in the verification set was $44.4 \%$. No statistical difference was found in the prevalence rate of WCH between data sets $(P=0.070$, Table 1$)$. Only gender and uric acid were statistically different between the training set and the verification set $(P=0.043, P=0.001$, Table 1$)$, and other modeling parameters were not statistically different between groups.

In the training set, the prevalence of ISH in WCH patients was significantly higher than that in SHT patients $(P<0.001$, Table 2). SBP, DBP and TG levels in SHT patients were significantly higher than those in the WCH group $(P=0.005, P<0.001, P=0.021$, Table 2$)$, and the prevalence rate of CCVD in SHT patients was significantly higher than that in WCH patients $(P=0.037$, Table 2$)$.

In the verification set, the prevalence rate of ISH in WCH patients was significantly higher than that in SHT patients, and SBP and DBP was significantly lower than that in SHT patients $(P=0.031, P=0.001, P=0.010$, Table 2). Besides, the proportion of females in $\mathrm{WCH}$ patients was significantly higher than that in SHT patients $(P=0.016$, Table 2$)$.

\section{Variable screening}

In LASSO regression screening, the corresponding screening variables were ISH, SBP, DBP, TG, Scr, and CCVD (Figure 1). Univariate logistic regression analysis in the training set showed that the above six parameters had statistical differences between WCH patients and SHT patients $(P<0.05$, Table 3), which 
should be included in the next multivariate regression modeling. The area under ROC curve (AUC) of ISH, SBP, DBP, TG, Scr and CCVD were 0.608, 0.581, 0.652, 0.566, 0.555 and 0.549 respectively (Table 3).

\section{Model construction and nomogram drawing}

Multivariate logistic regression was used to construct the scoring model and draw the nomogram. In clinical application, ISH and other variables can be scored one by one, and the total score can be calculated after addition. The possibility of WCH in patients with elevated office blood pressure can be known by comparing with the nomogram (Figure 2).

\section{Discrimination degree and calibration degree}

The discrimination degree of the scoring model had statistical significance in both the training set and the verification set $(P<0.001, P<0.001$, Table 4$)$. The $\mathrm{R}^{2}$ and AUC of the scoring model in the training set were 0.163 and 0.705 (95\% Cl: 0.656-0.754), respectively. In the verification set, the $\mathrm{R}^{2}$ of the scoring model was 0.206, and AUC was 0.718 (95\% Cl: 0.621-0.814). The model had a certain discrimination degree in both the training set and the verification set, and the discrimination degree in the training set and the verification set was close.

The calibration test results showed that the scoring model had good stability in both training set and verification set $(\mathrm{MSE}=0.001, \mathrm{MAE}=0.014 \mathrm{MSE}=0.001, \mathrm{MAE}=0.025$, respectively, Table 4, Figure 3).

\section{Participation rate and missed diagnosis rate of hypertension guideline method and scoring model method}

According to the practice guide method for hypertension, 499 of 553 patients with elevated office blood pressure needed ambulatory blood pressure monitoring, with a participation rate of $90.2 \%$. 427 patients needed ambulatory blood pressure monitoring if the scoring model method was used. The participation rate was $77.2 \%$. There was a statistical difference in the participation rate between the two methods ( $P]$ 0.001 , Table 5). However, the missed diagnosis rate of $\mathrm{WCH}$ caused by the hypertension practice guide method was $6.8 \%$, and that of the scoring model method was $7.8 \%$. There was no statistical difference in the missed diagnosis rate $(P=0.433$, Table 5$)$.

\section{Discussion}

How to identify WCH high-risk patients during initial diagnosis is currently a clinical problem. At present, some clinicians empirically distinguish WCH from SHT by combining $\mathrm{WCH}$ with the clinical characteristics of the high incidence of ISH, insignificant damage to target organs, and SHT with high incidence in men and smokers and close relationship with blood lipid and kidney function [14, 32]. However, it is impossible to judge the risk of $\mathrm{WCH}$ scientifically and quantitatively. At present, there is still a lack of a scientific scoring model for differential diagnosis of WCH and SHT. In this study, we constructed this scoring model for differential diagnosis of WCH and SHT. 
In this modeling process, LASSO regression analysis is first selected to screen research variables. LASSO regression is a relatively accurate model variable screening method at present. Compared with other variable screening methods such as ridge regression, LASSO regression can not only screen variables but also adjust complexity, effectively avoiding model overfitting [33]. In this study, 17 research variables such as gender and age were included into the LASSO regression model. Including general information such as sex and age, office blood pressure, and biochemical test results such as blood lipid and renal function, the candidate variables are all clinically readily available indicators. According to the clinical characteristics of $\mathrm{WCH}$, which is high incidence in ISH patients, ISH is divided according to the characteristics of office blood pressure and included in the election variables. Blood glucose-related variables are included in DM instead of fasting blood glucose, while blood lipid variables are included in TC, TG, HDL-C, and LDL-C test results instead of hyperlipidemia. The main consideration is that blood lipid variables are more complex and have different representative meanings, so they are listed separately. Scr and UA are the most common but clinically significant renal function indicators, which are also included in the variables to be screened.

A total of six variables participating in the model construction were screened by LASSO regression, namely ISH, SBP, DBP, TG, Scr, and CCVD. These six items reflect the clinical characteristics of individual blood pressure, blood lipid, renal function, and target organ injury. For a long time, ISH has believed that it is closely related to $\mathrm{WCH}$. In 2019, Feitosa et al. confirmed once again that WCH has the clinical feature of increasing ISH proportion at different age groups [14]. The results of this LASSO regression study also suggest that ISH is positively correlated with WCH. ARTEMIS research involving 27 countries around the world found that SHT is more common than WCH in patients with hyperlipidemia and renal insufficiency [34]. In this study, we also screened that TG and Scr are both positively correlated with SHT. The selected variables in blood lipid index are TG, not TC, and LDL. We speculate that the reason may be that some participants have used lipid-lowering drugs. At present, statins are the most widely used lipid-lowering drugs, and their significant reduction of TC and LDL may interfere with the research results [35]. TG is less affected by statins, so it is more suitable to be included in the scoring model.

European Hypertension Practice Guide (2018 Edition) suggests that people with grade 1 hypertension or elevated office blood pressure without obvious target organ damage should be suspected to have white coat hypertension, and further ambulatory blood pressure monitoring should be conducted to make a definite diagnosis [31]. In other words, it is based on the blood pressure level and target organ damage to judge the possibility of white coat hypertension.

The scoring model is actually an improvement of the European Hypertension Guidelines. Finally $₫$ we can not only rely on SBP and DBP (reflecting blood pressure of participants) and CCVD information but also combine other parameters. Firstly, the scoring model reflects the clinical characteristics of a high proportion of ISH in white coat hypertension, and also reflects the clinical characteristics of relatively high TG and Scr in SHT. 
Finally, this study uses ISH, SBP, DBP, TG, Scr, and CCVD to build a differential diagnosis model in the training set. The results suggest that ISH, SBP, DBP, TG, Scr, and CCVD alone are used to distinguish WCH from SHT and AUC are $0.608,0.581,0.652,0.566,0.555$ and 0.549 respectively, and the discrimination ability is low. However, the construction of the differential diagnosis model can improve the discrimination ability of WCH. The AUC of the model applied to the training set and the verification set are 0.705 and 0.718 respectively, which is significantly improved compared with the ability of univariate WCH identification. Moreover, the AUC values of the training set and the verification set are relatively close, which indicates that the model is relatively robust [36]. Further calibration test results show that the model has good stability in both the training set and verification set.

The clinical application of this model can transform the previous empirical method of distinguishing WCH into a scientific clinical tool that can quantitatively predict the discovery of WCH diseases. Also, the model has one important use. The European Hypertension Practice Guidelines only point out that ambulatory blood pressure monitoring should be carried out for patients with grade 1 hypertension and hypertensive patients without target organ damage to identify $\mathrm{WCH}$. However, the model selected participants with WCH risk higher than $20 \%$ to monitor ambulatory blood pressure. Compared with the two methods, the differential diagnosis model can significantly reduce the number of participants and save corresponding medical expenses and time loss while maintaining a similar missed diagnosis rate.

In this study, we innovatively try to distinguish WCH from SHT by scoring model, and the established model has certain clinical value. This has two disadvantages. Firstly, The training set and verification sets are randomly divided according to our own research data. The verification method used is internal verification, and the application value of the model needs a large sample of external data for verification. In addition, this model is still limited to people with normal renal function, and people with renal insufficiency cannot apply this model, so there are some limitations in its application.

\section{Conclusion}

In a word, this study established a scoring model for differential diagnosis between WCH and SHT in people with normal renal function. The scoring model has good stability and a certain discrimination degree. It can scientifically and quantitatively evaluate the possibility of $\mathrm{WCH}$ at the beginning of diagnosis, which is helpful to identify WCH in clinical practice.

\section{Abbreviations}

WCH, white coat hypertension; SHT, sustained hypertension; BMI, body mass index; SBP, office systolic blood pressure; DBP, office diastolic blood pressure; TC, total cholesterol; TG, triglyceride; HDL-C, highdensity lipoprotein cholesterol; LDL-C, low-density lipoprotein cholesterol; Glu, fasting blood glucose; Scr, serum creatinine; UA, serum uric acid; ISH, isolated systolic hypertension; CCVD, cardiovascular and cerebrovascular diseases; LASSO regression, the least absolute shrinkage and selection operator 
regression; $R^{2}$, the coefficient of determination; OR, odds ratio; ROC, receiver operating curve; $A U C$, areas under the ROC curve; MSE, mean square error; MAE, mean absolute error.

\section{Declarations}

\section{Ethics approval and consent to participate}

The study was reviewed and approved by the Ethics Committee of Daping Hospital of the Army Medical University and registered in the Chinese Clinical Trial Registry with the registration number ChiCTR1800015507. All participants gave informed consent to the study.

\section{Consent for publication}

Not applicable.

\section{Availability of data and materials}

In attempt to preserve the privacy of individuals, clinical data will not be shared; the data can be available from authors upon request.

\section{Authors' contributions}

Peng Cai, Yan Wang, Xukai Wang: contributed to the study design. Peng Cai, Yan Peng, YuXi Chen: performed the data collection and analyses, and drafted the paper. All authors read and approved the final manuscript.

\section{Competing interests}

The authors declare that they have no actual or potential conflicts of interest.

\section{Acknowledgments}

May my son Domi grow up healthily.

\section{Funding}

The National Natural Science Foundation of China (No. 81860643) supported the work.

\section{Disclosure of conflict of interest}

The authors declare that they have no actual or potential conflicts of interest

\section{References}


1. Abolbashari M: White Coat Hypertension and Cardiovascular Diseases: Innocent or Guilty. Curr Cardiol Rep 2018, 20:25.

2. Cai $P$, Zhong W, Wang $Y$, Wang X: Effects of white-coat, masked and sustained hypertension on coronary artery stenosis and cardiac arrhythmia. Hypertens Res 2020, 43:121-131.

3. Sivén SS, Niiranen TJ, Kantola IM, Jula AM: White-coat and masked hypertension as risk factors for progression to sustained hypertension: the Finn-Home study. J Hypertens 2016, 34:54-60.

4. Zhang ZY, Asayama K, Franklin SS, Thijs L, Staessen JA: Cardiovascular Events and Mortality in White Coat Hypertension. Ann Intern Med 2019, 171:602-603.

5. Cai P, Peng Y, Wang Y, Wang X: Effect of white-coat hypertension on arterial stiffness: A metaanalysis. Medicine (Baltimore) 2018, 97:e12888.

6. Franklin SS, Thijs L, Asayama K, Li Y, Hansen TW, Boggia J, Jacobs L, Zhang Z, Kikuya M, BjörklundBodegård K, et al: The Cardiovascular Risk of White-Coat Hypertension. J Am Coll Cardiol 2016, 68:2033-2043.

7. de la Sierra A, Vinyoles E, Banegas JR, Segura J, Gorostidi M, de la Cruz JJ, Ruilope LM: Prevalence and clinical characteristics of white-coat hypertension based on different definition criteria in untreated and treated patients. J Hypertens 2017, 35:2388-2394.

8. Bloomfield DA, Park A: Decoding white coat hypertension. World J Clin Cases 2017, 5:82-92.

9. Muxfeldt ES, Bloch KV, Nogueira Ada R, Salles GF: True resistant hypertension: is it possible to be recognized in the office? Am J Hypertens 2005, 18:1534-1540.

10. O'Brien E, White WB, Parati G, Dolan E: Ambulatory blood pressure monitoring in the 21 st century. $J$ Clin Hypertens (Greenwich) 2018, 20:1108-1111.

11. Stergiou GS, Kario K, Kollias A, McManus RJ, Ohkubo T, Parati G, Imai Y: Home blood pressure monitoring in the 21st century. J Clin Hypertens (Greenwich) 2018, 20:1116-1121.

12. Kinsara AJ: Ambulatory blood pressure monitoring in daily practice. Indian Heart J 2017, 69:788-789.

13. Shimbo D, Muntner P: Should Out-of-Office Monitoring Be Performed for Detecting White Coat Hypertension? Ann Intern Med 2019, 170:890-892.

14. Feitosa ADM, Mota-Gomes MA, Barroso WS, Miranda RD, Barbosa ECD, Pedrosa RP, Oliveira PC, Feltosa C, Brandão AA, Lima-Filho JL, et al: Relationship between office isolated systolic or diastolic hypertension and white-coat hypertension across the age spectrum: a home blood pressure study. $J$ Hypertens 2020, 38:663-670.

15. Cuspidi C, Sala C, Tadic M, Rescaldani M, Grassi G, Mancia G: Is white-coat hypertension a risk factor for carotid atherosclerosis? A review and meta-analysis. Blood Press Monit 2015, 20:57-63.

16. Yang J, Pan Z, Zhao F, Feng X, Liu Q, Li Y, Lyu J: A nomogram for predicting survival in patients with nodular melanoma: A population-based study. Medicine (Baltimore) 2019, 98:e16059.

17. Cantinotti M, Giordano R, Scalese M, Molinaro S, Murzi B, Assanta N, Crocetti M, Marotta M, Ghione $S$, lervasi G: Strengths and limitations of current pediatric blood pressure nomograms: a global 
overview with a special emphasis on regional differences in neonates and infants. Hypertens Res 2015, 38:577-587.

18. Jia XY, Yu JT, Hu SY, Li JN, Wang M, Wang C, Chen M, Cui Z, Zhao MH: Antibodies against linear epitopes on Goodpasture autoantigen in patients with anti-neutrophil cytoplasmic antibodyassociated vasculitis. Clin Rheumato/ 2017, 36:2087-2094.

19. Kinder F, Giannoudis PV, Boddice T, Howard A: The Effect of an Abnormal BMI on Orthopaedic Trauma Patients: A Systematic Review and Meta-Analysis. J Clin Med 2020, 9.

20. Park SH, Park YS: Can an automatic oscillometric device replace a mercury sphygmomanometer on blood pressure measurement? a systematic review and meta-analysis. Blood Press Monit 2019, 24:265-276.

21. Parati G, Stergiou G, O'Brien E, Asmar R, Beilin L, Bilo G, Clement D, de la Sierra A, de Leeuw P, Dolan E, et al: European Society of Hypertension practice guidelines for ambulatory blood pressure monitoring. J Hypertens 2014, 32:1359-1366.

22. Cai P, Peng Y, Chen Y, Li L, Chu W, Wang Y, Wang X: Association of thyroid function with white coat hypertension and sustained hypertension. J Clin Hypertens (Greenwich) 2019, 21:674-683.

23. Bayó J, Dalfó A, Barceló MA, Saez M, Roca C, Pallozzi J, Coll-De-Tuero G: The Role First-Day Readings Play in a 3-Day Schedule of Self-Monitoring Home Blood Pressure Based on Prognostic Data. VAMPAHICA Study. Am J Hypertens 2020, 33:154-160.

24. Wei Y, Xu Q, Yang H, Yang Y, Wang L, Chen H, Anderson C, Liu X, Song G, Li Q, et al: Preconception diabetes mellitus and adverse pregnancy outcomes in over 6.4 million women: A population-based cohort study in China. PLoS Med 2019, 16:e1002926.

25. Berry KJ, Mielke PW, Jr.: Exact and Monte carlo resampling procedures for the Wilcoxon-MannWhitney and Kruskal-Wallis tests. Percept Mot Skills 2000, 91:749-754.

26. Hessam S, Scholl L, Sand M, Schmitz L, Reitenbach S, Bechara FG: A Novel Severity Assessment Scoring System for Hidradenitis Suppurativa. JAMA Dermatol 2018, 154:330-335.

27. Alhamzawi R, Ali HTM: The Bayesian adaptive lasso regression. Math Biosci 2018, 303:75-82.

28. Park SY: Nomogram: An analogue tool to deliver digital knowledge. J Thorac Cardiovasc Surg 2018, 155:1793.

29. Tammemägi MC, Katki HA, Hocking WG, Church TR, Caporaso N, Kvale PA, Chaturvedi AK, Silvestri GA, Riley TL, Commins J, Berg CD: Selection criteria for lung-cancer screening. N Engl J Med 2013, 368:728-736.

30. Hussain L, Saeed S, Idris A, Awan IA, Shah SA, Majid A, Ahmed B, Chaudhary QA: Regression analysis for detecting epileptic seizure with different feature extracting strategies. Biomed Tech (Berl) 2019, 64:619-642.

31. Williams B, Mancia G, Spiering W, Agabiti Rosei E, Azizi M, Burnier M, Clement D, Coca A, De Simone G, Dominiczak A, et al: 2018 Practice Guidelines for the management of arterial hypertension of the European Society of Cardiology and the European Society of Hypertension. Blood Press 2018, 27:314-340. 
32. Thomas O, Shipman KE, Day K, Thomas M, Martin U, Dasgupta I: Prevalence and determinants of white coat effect in a large UK hypertension clinic population. J Hum Hypertens 2016, 30:386-391.

33. Huang JC, Tsai YC, Wu PY, Lien YH, Chien CY, Kuo CF, Hung JF, Chen SC, Kuo CH: Predictive modeling of blood pressure during hemodialysis: a comparison of linear model, random forest, support vector regression, XGBoost, LASSO regression and ensemble method. Comput Methods Programs Biomed 2020, 195:105536.

34. Omboni S, Aristizabal D, De la Sierra A, Dolan E, Head G, Kahan T, Kantola I, Kario K, Kawecka-Jaszcz K, Malan L, et al: Hypertension types defined by clinic and ambulatory blood pressure in 14143 patients referred to hypertension clinics worldwide. Data from the ARTEMIS study. J Hypertens 2016, 34:2187-2198.

35. Ai C, Zhang S, He Q, Shi J: Comparing the combination therapy of ezetimibe and atorvastatin with atorvastatin monotherapy for regulating blood lipids: a systematic review and meta-analyse. Lipids Health Dis 2018, 17:239.

36. Zhang J, Pan Z, Yang J, Yan X, Li Y, Lyu J: A nomogram for determining the disease-specific survival in Ewing sarcoma: a population study. BMC Cancer 2019, 19:667.

\section{Tables}


Table 1.

Statistical analysis of the differences of research parameters between training set and verification set.

\begin{tabular}{|c|c|c|c|}
\hline Parameters & Training set & Validation set & $P$ value \\
\hline Individuals & 445 & 108 & - \\
\hline WCH (\%) & $156(35.1)$ & $48(44.4)$ & 0.070 \\
\hline Female $\% \rrbracket$ & $191(42.9)$ & $58(53.7)$ & 0.043 \\
\hline 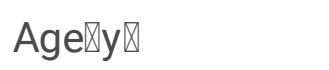 & $64.0(18.0)$ & $65.0(16.0)$ & 0.734 \\
\hline BMI (kg/m2) & $24.2(4.4)$ & $23.8(3.5)$ & 0.061 \\
\hline Smoking (\%) & $143(32.1)$ & $31(28.7)$ & 0.491 \\
\hline Drinking (\%) & $109(24.5)$ & $27(25.0)$ & 0.913 \\
\hline Diabetes (\%) & 69 (15.5) & $16(14.8)$ & 0858 \\
\hline ISH (\%) & $220(49.4)$ & 55 (50.9) & 0.781 \\
\hline SBP & $144.0(15.0)$ & $145.0(12.0)$ & 0.347 \\
\hline DBP & $90.0(14.0)$ & $88.5(15.0)$ & 0.894 \\
\hline $\mathrm{HR}$ & $78.0(18.0)$ & 77.5 (18.0) & 0.819 \\
\hline $\mathrm{TC}(\mathrm{mmol} / \mathrm{L})$ & $4.24(1.37)$ & $4.16(1.28)$ & 0.435 \\
\hline $\mathrm{TG}(\mathrm{mmol} / \mathrm{L})$ & $1.42(1.05)$ & $1.27(0.75)$ & 0.096 \\
\hline $\mathrm{HDL}-\mathrm{C}(\mathrm{mmol} / \mathrm{L})$ & $1.09(0.35)$ & $1.11(0.37)$ & 0.563 \\
\hline LDL-C (mmol/L) & $2.71(1.01)$ & $2.58(0.93)$ & 0.490 \\
\hline $\operatorname{Scr}(\mu \mathrm{mol} / \mathrm{L})$ & $66.4(21.1)$ & $63.9(22.7)$ & 0.217 \\
\hline $\mathrm{UA}(\mu \mathrm{mol} / \mathrm{L})$ & 337.2 (121.6) & $303.5(98.9)$ & 0.001 \\
\hline CCVD (\%) & $154(34.6)$ & $46(42.6)$ & 0.121 \\
\hline
\end{tabular}

WCH, white coat hypertension; BMI, body mass index; SBP, office systolic blood pressure; DBP, office diastolic blood pressure; TC, total cholesterol; TG, triglyceride; HDL-C, high-density lipoprotein cholesterol; LDL-C, low-density lipoprotein cholesterol; Glu, fasting blood glucose; Scr, serum creatinine; UA, serum uric acid; ISH, isolated systolic hypertension; CCVD, cardiovascular and cerebrovascular diseases. 
Table 2.

Statistical analysis of the differences of research variables between WCH patients and SHT patients.

\begin{tabular}{|c|c|c|c|c|c|c|}
\hline \multirow[t]{2}{*}{ Parameters } & \multicolumn{2}{|l|}{ Training set } & \multirow{2}{*}{$\begin{array}{l}P \\
\text { value }^{*}\end{array}$} & \multicolumn{2}{|c|}{ Validation set } & \multirow{2}{*}{$\begin{array}{l}P \\
\text { value }^{\#}\end{array}$} \\
\hline & WCH & SHT & & WCH & SHT & \\
\hline Individuals & 156 & 289 & - & 48 & 60 & - \\
\hline Female $\% \rrbracket$ & $70(44.9)$ & 121 (41.9) & 0.541 & $32(66.7)$ & $26(43.3)$ & 0.016 \\
\hline Age $₫ y \rrbracket$ & $64.0(14.0)$ & $64.0(18.0)$ & 0.716 & $65.0(17.0)$ & $64.5(15.0)$ & 0.805 \\
\hline BMI $\left(\mathrm{kg} / \mathrm{m}^{2}\right)$ & $24.5(4.5)$ & $24.2(4.4)$ & 0.471 & $23.6(3.8)$ & 23.9 (3.5) & 0.257 \\
\hline Smoking (\%) & $42(26.9)$ & $101(22.7)$ & 0.084 & $11(22.9)$ & 20 (33.3) & 0.234 \\
\hline Drinking (\%) & $41(24.5)$ & $68(23.5)$ & 0.519 & $7(14.6)$ & $20(33.3)$ & 0.025 \\
\hline Diabetes (\%) & $21(13.5)$ & $48(16.6)$ & 0.381 & $6(12.5)$ & $10(16.7)$ & 0.545 \\
\hline ISH (\%) & $99(63.5)$ & 121 (41.9) & $<0.001$ & $30(62.5)$ & $25(41.7)$ & 0.031 \\
\hline SBP & $142.5(14.0)$ & $145.0(17.0)$ & 0.005 & $142.5(7.0)$ & $\begin{array}{l}148.0 \\
(18.0)\end{array}$ & 0.001 \\
\hline DBP & $83.0(13.0)$ & $90.0(12.0)$ & $<0.001$ & $84.5(13.0)$ & $91.0(17.0)$ & 0.010 \\
\hline $\mathrm{HR}$ & $78.0(20.0)$ & $79.0(15.0)$ & 0.398 & $76.0(18.0)$ & $78.0(18.0)$ & 0.204 \\
\hline TC (mmol/L) & $4.23(1.28)$ & $4.29(1.43)$ & 0.277 & $4.16(1.50)$ & $4.18(1.11)$ & 0.936 \\
\hline TG (mmol/L) & $1.52(1.20)$ & $1.34(0.83)$ & 0.021 & $1.26(0.94)$ & $1.27(0.74)$ & 0.483 \\
\hline $\begin{array}{l}\mathrm{HDL}-\mathrm{C} \\
(\mathrm{mmol} / \mathrm{L})\end{array}$ & $1.12(0.35)$ & $1.08(0.35)$ & 0.272 & $1.14(0.38)$ & $1.09(0.35)$ & 0.483 \\
\hline $\begin{array}{l}\text { LDL-C } \\
(\mathrm{mmol} / \mathrm{L})\end{array}$ & $2.70(0.92)$ & $2.75(1.07)$ & 0.293 & $2.59(0.92)$ & $2.59(1.02)$ & 0.850 \\
\hline $\operatorname{Scr}(\mu \mathrm{mol} / \mathrm{L})$ & 64.6 (19.3) & $67.1(21.2)$ & 0.053 & $59.6(20.0)$ & $67.3(23.2)$ & 0.187 \\
\hline $\mathrm{UA}(\mu \mathrm{mol} / \mathrm{L})$ & $\begin{array}{l}328.7 \\
(112.6)\end{array}$ & $\begin{array}{l}346.8 \\
(124.9)\end{array}$ & 0.050 & $\begin{array}{l}297.6 \\
(87.4)\end{array}$ & $\begin{array}{l}313.1 \\
(109.3)\end{array}$ & 0.130 \\
\hline CCVD (\%) & $44(28.2)$ & $110(38.1)$ & 0.037 & $16(33.3)$ & $30(50.0)$ & 0.082 \\
\hline
\end{tabular}

$\mathrm{WCH}$, white coat hypertension; SHT, sustained hypertension; BMI, body mass index; SBP, office systolic blood pressure; DBP, office diastolic blood pressure; TC, total cholesterol; TG, triglyceride; HDL-C, highdensity lipoprotein cholesterol; LDL-C, low-density lipoprotein cholesterol; Glu, fasting blood glucose; Scr, serum creatinine; UA, serum uric acid; ISH, isolated systolic hypertension; CCVD, cardiovascular and cerebrovascular disease. 
Table 3.

Univariate logistic regression analysis of the research variables participating in the model construction and the results of each discrimination test.

\begin{tabular}{|llll|}
\hline Parameters & \multicolumn{2}{l}{ Univariate logistic regression } & AUC (95\% Cl) \\
& OR (95\%Cl) & $P$ value & \\
\hline ISH & $2.411(1.615-3.601)$ & $\otimes 0.001$ & $0.608(0.553-0.663)$ \\
\hline SBP & $0.977(0.964-0.992)$ & 0.002 & $0.581(0.526-0.635)$ \\
DBP & $0.957(0.939-0.974)$ & $\otimes 0.001$ & $0.652(0.599-0.704)$ \\
\hline TG & $0.811(0.687-0.957)$ & 0.013 & $0.566(0.512-0.621)$ \\
\hline Scr & $0.987(0.975-0.999)$ & 0.029 & $0.555(0.500-0.611)$ \\
\hline CCVD & $0.639(0.419-0.975)$ & 0.038 & $0.549(0.494-0.605)$ \\
\hline
\end{tabular}

SBP, office systolic blood pressure; DBP, office diastolic blood pressure; TG, triglyceride; Scr, serum creatinine; ISH, isolated systolic hypertension; CCVD, cardiovascular and cerebrovascular diseases; AUC, areas under the ROC curve; OR, odds ratio.

Table 4.

Univariate logistic regression analysis of the research variables participating in the model construction and the results of each discrimination test.

\begin{tabular}{|lllllll|}
\hline Data sets & Samples & $\mathbf{R}^{2}$ & AUC $(95 \% \mathrm{Cl})$ & $P$ value & MSE & MAE \\
\hline Training set & 445 & 0.163 & $0.705(0.656-0.754)$ & $\varangle 0.001$ & $\varangle 0.001$ & 0.014 \\
\hline Validation set & 108 & 0.206 & $0.718(0.621-0.814)$ & $\varangle 0.001$ & 0.001 & 0.025 \\
\hline
\end{tabular}

$\mathrm{R}^{2}$, the coefficient of determination; AUC, areas under the ROC curve; MSE, mean square error; MAE, mean absolute error.

Table 5.

The difference between the participation rate of ambulatory blood pressure monitoring in outpatients with hypertension and the missed diagnosis rate of WCH patients between the hypertension practice guideline method and the scoring model method.

\begin{tabular}{|lllllll|}
\hline Methods & $\begin{array}{l}\text { Total } \\
\text { individuals } \\
\text { (\%) }\end{array}$ & $\begin{array}{l}\text { Checked } \\
\text { Individuals } \\
(\%)\end{array}$ & $\begin{array}{l}\boldsymbol{P} \\
\text { value }^{*}\end{array}$ & $\begin{array}{l}\text { Total WCH } \\
\text { patients (\%) }\end{array}$ & $\begin{array}{l}\text { Missed WCH } \\
\text { patients (\%) }\end{array}$ & $\begin{array}{l}\boldsymbol{P} \\
\text { value }^{\#}\end{array}$ \\
\hline $\begin{array}{l}\text { Method } \\
1\end{array}$ & $553(100)$ & $499(90.2)$ & $\begin{array}{l}0 \\
0.001\end{array}$ & $204(100)$ & $12(6.8)$ & 0.433 \\
\hline $\begin{array}{l}\text { Method } \\
2\end{array}$ & $533(100)$ & $427(77.2)$ & & $204(100)$ & $16(7.8)$ & \\
\hline
\end{tabular}


$P$ value ${ }^{*}$ represents the statistical difference between groups in participation rate; $P$ value ${ }^{\#}$ represented the statistical difference of missed diagnosis rate between the groups. $\mathrm{WCH}$, white coat hypertension.

\section{Figures}

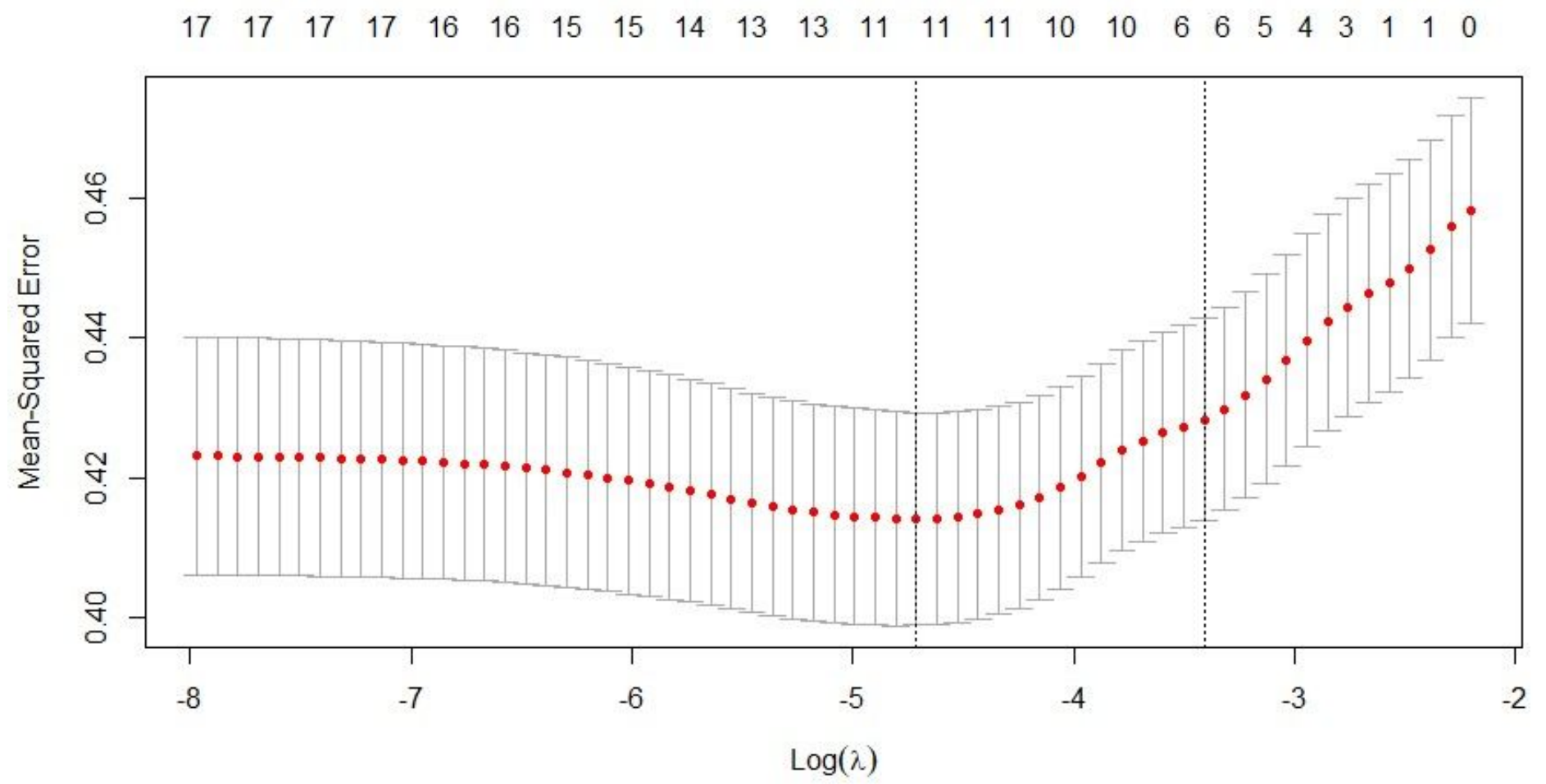

Figure 1

The result of LASSO regression analysis of variable screening in training set. LASSO regression was used to screen the research variables by 10 -fold cross-validation. The upper abscissa indicated the number of selected variables, while the left vertical dotted line indicates that 11 variables were selected when the minimum penalty parameter standard was adopted, while the right vertical dotted line indicates that the number of selected variables was 6 when one standard error (1-SE standard) was adopted. 
Points

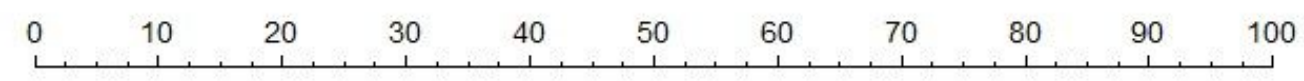

ISH

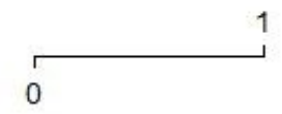

SBP

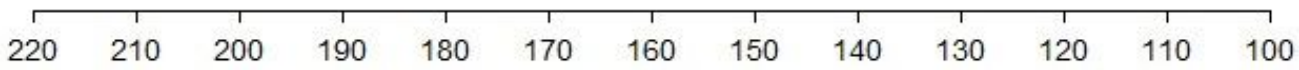

DBP

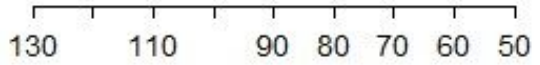

TG

$\begin{array}{rrrrrrrrrrr}10 & 9 & 8 & 7 & 6 & 5 & 4 & 3 & 2 & 1 & 0\end{array}$

Scr

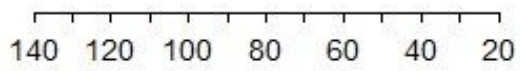

CCVD

0

1

Total Points

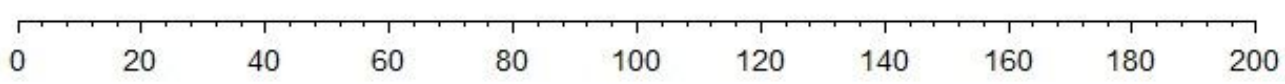

WCH

$\begin{array}{lllllllllll}0.01 & 0.05 & 0.1 & 0.2 & 0.3 & 0.4 & 0.5 & 0.6 & 0.7\end{array}$

\section{Figure 2}

The nomogram for differential diagnosis of WCH and SHT. Clinicians scored ISH and other parameters one by one with reference to the nomogram, and the total score could be calculated after addition. By comparing with nomogram, we could know the possibility of WCH in patients with elevated office blood pressure. 


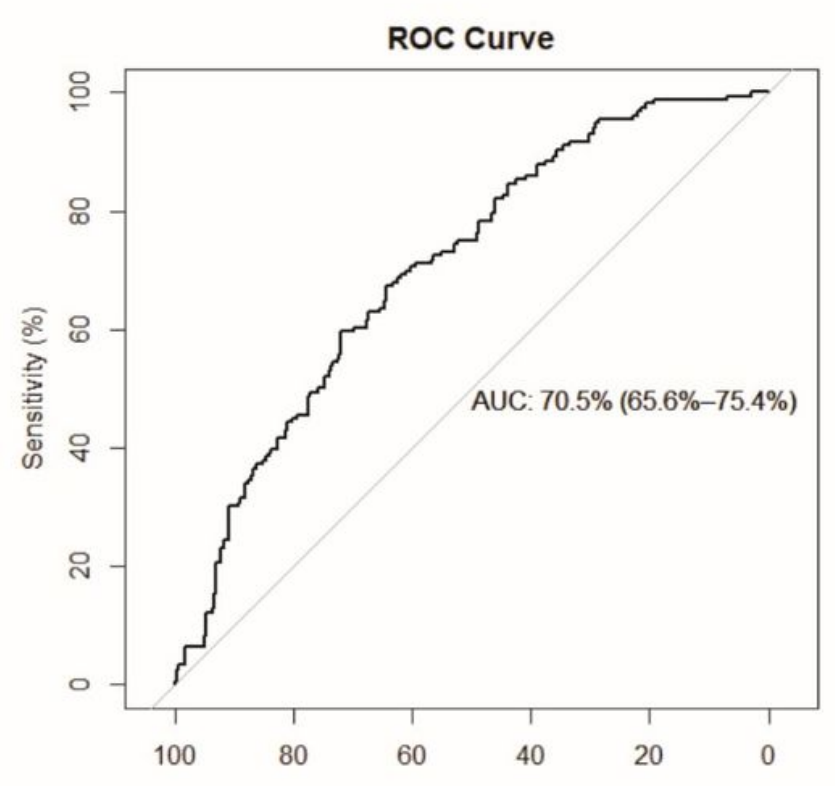

Calibration Curve

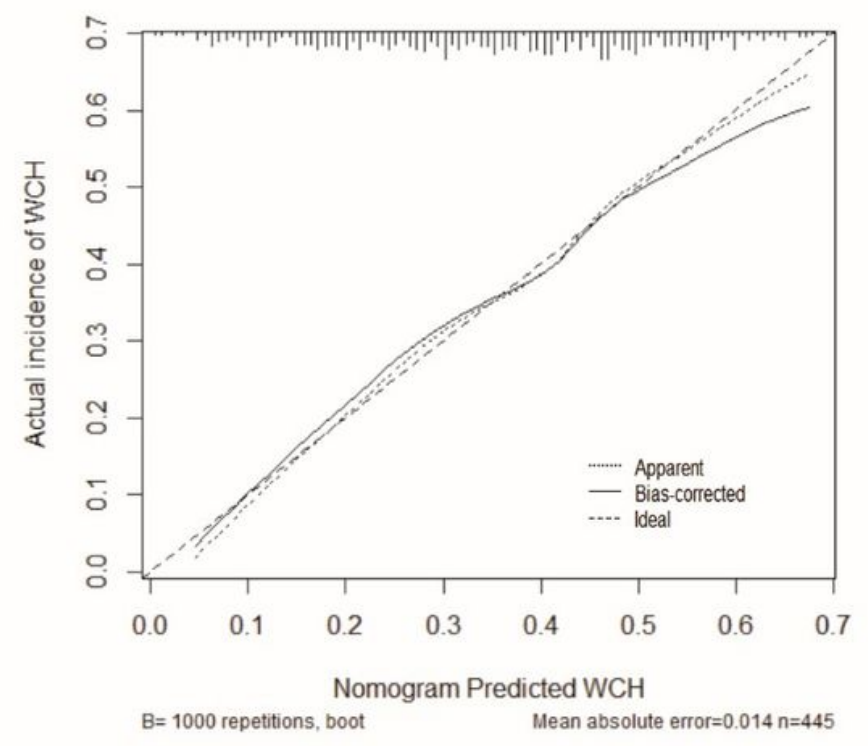

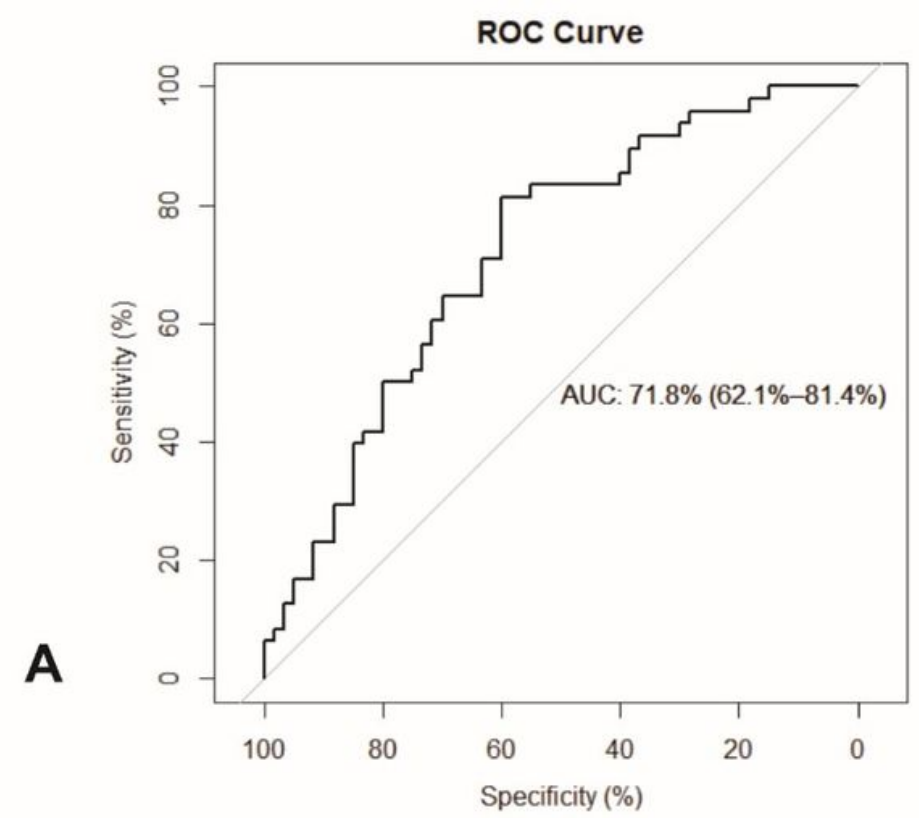

B

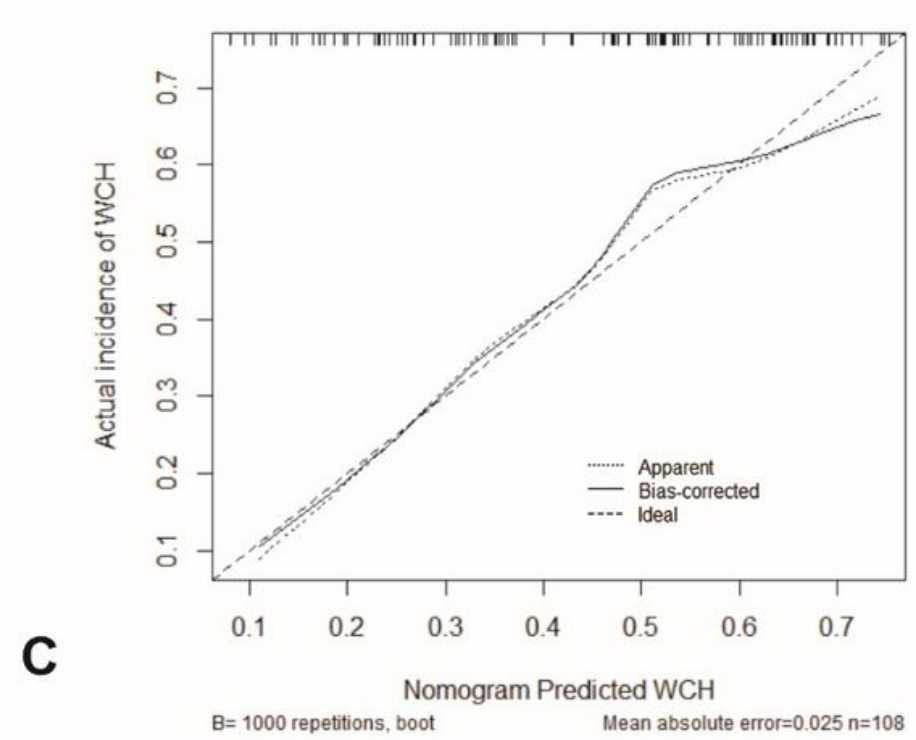

\section{Figure 3}

Discrimination and calibration test of the scoring model. Figure $A$ and Figure $B$ show the discrimination of the scoring model in the training set and the verification set, respectively. Figure $C$ and Figure $D$ suggest the calibration degree of the scoring model in the training set and the verification set respectively.

\section{Supplementary Files}

This is a list of supplementary files associated with this preprint. Click to download.

- DatainBrief.rar 